\title{
Fecundidade abaixo da reposição, população estacionária por migração e efeitos sobre a estrutura etária
}

\author{
André Junqueira Caetano*
}

\begin{abstract}
Diante do declínio da fecundidade, o Brasil aproxima-se das condições demográficas que determinam o crescimento zero ou negativo, no longo prazo, de uma população. Entretanto, não há razões suficientes para se afirmar que a fecundidade estabilizar-se-á no nível de reposição ou pouco abaixo dele. Há indicações de que ela deve atingir patamares bastante baixos a esse nível nas próximas décadas. Teoricamente, uma população estável e fechada com taxas de fecundidade abaixo do nível de reposição pode retomar o crescimento zero se submetida a uma mudança do número anual constante de entradas derivadas do retorno da fecundidade ao nível de reposição. Isto também pode ocorrer devido a saldos líquidos migratórios positivos, constantes e com estrutura etária fixa. Portanto, para um determinado conjunto de taxas de saída existe um número infinito de populações estacionárias equivalentes. Pressupondo um regime de fecundidade abaixo da reposição, este trabalho utiliza o modelo teórico de Schmertmann (1992) para simular e examinar, comparativamente, a estrutura etária da população do Estado de São Paulo, resultante de um aumento da fecundidade até o nível de reposição, e as estruturas etárias desta mesma população tornada estacionária via migração, assumindo diferentes cenários de fecundidade e estrutura etária das imigrantes.
\end{abstract}

Palavras-chave: Migração. Fecundidade abaixo da reposição. Estrutura etária. Envelhecimento populacional.

\section{Introdução}

O declínio da fecundidade para níveis inferiores ao de reposição tem suscitado debates e análises, principalmente nos países onde este componente atingiu patamares bastante baixos, como é o caso de vários países da Europa Ocidental (FEICHTINGER; STEINMANN, 1992). Tal decréscimo tem rebatimento direto e substancial sobre duas tendências demográficas de extrema importância: a diminuição e o envelhecimento populacional.

Diante deste quadro, acrescido dos ganhos de mortalidade em idades mais avan- çadas (CAMPOS; RODRIGUES, 2004), uma questão que tem sido levantada relaciona-se ao papel da migração - e, nos casos dos países com fecundidade já muito baixa, da migração internacional - no sentido de interromper o declínio populacional em populações com fecundidade abaixo da taxa de reposição. O papel da migração para contrabalançar os efeitos da fecundidade abaixo da reposição é também denominado de reposição e refere-se basicamente a fluxos internacionais (NAÇÕES UNIDAS, 2000).

De acordo como dados do Censo Demográfico 2000, a Taxa de Fecundidade

\footnotetext{
* Professor da PUC Minas, Programa de Pós-graduação em Ciências Sociais.
} 
Total (TFT) brasileira, naquele ano, era de 2,4 filhos por mulher, apresentando uma diversidade ainda importante entre as macrorregiões e os estados. Na Região Norte, a TFT era de 3,2, enquanto no Sudeste, já atingia o limiar da reposição, isto é, 2,1 filhos por mulher. Portanto, na Região Sudeste, era de se esperar que algumas de suas áreas apresentassem, em 2000 , níveis abaixo da reposição. De fato, este é o caso de São Paulo, cuja TFT estava em 2,0 no conjunto do estado, com diversas de suas regiões apresentando índices inferiores a 1,8 (CAMARGO; YAZAKI, 2002).

As inter-relações entre migração e fecundidade e seus efeitos sobre a estrutura etária em uma população com fecundidade abaixo da reposição vêm sendo analisadas por vários autores (ESPENSHADE et al., 1982; MITRA, 1983 e 1990). Neste aspecto, o trabalho seminal de Schmertmann (1992) estabelece que qualquer população com este nível de fecundidade e com uma estrutura etária de imigrantes fixa ao longo do tempo alcança a estacionariedade e, conseqüentemente, retorna à taxa de crescimento zero. Ademais, qualquer população com fecundidade abaixo do nível de reposição que retome o crescimento zero por meio da migração terá uma estrutura etária relativamente mais envelhecida do que a população tornada estacionária por intermédio do aumento da fecundidade até o nível de reposição.

O objetivo deste artigo é efetuar um exercício de simulação, no qual o Estado de São Paulo será o "país" de referência e o restante do Brasil será o "resto do mundo", de forma a se estimarem as estruturas etárias resultantes de diferentes cenários da fecundidade e de migração. Especificamente, utiliza-se o método de projeção dos componentes, tomando-se os saldos migratórios por idade observados no qüinqüênio 1995-2000 como a estrutura de entrada de imigrantes do "mundo" para este estado. Nos grupos etários em que o saldo migratório observado foi negativo, a migração foi considerada nula. O procedimento utilizado neste artigo considera, portanto, apenas entradas via migração, ou seja, não há emigração. Esta opção justifica-se pelo fato de já se usar, para os grupos com saldo positivo, a diferença entre entradas e saídas via migração. ${ }^{1}$

Os cenários de fecundidade construídos são os seguintes: (a) fecundidade observada em 2000 (TFT de 2,04) e ausência de imigração; (b) retomada da fecundidade ao nível de reposição e ausência de imigração; (c) fecundidade observada em $2000(2,04)$ e imigração por idade correspondente ao saldo migratório feminino paulista observado; (d) menor fecundidade entre as regiões administrativas de São Paulo em 2001 (1,55, na RA de São José do Rio Preto) ${ }^{2}$ e ausência de imigração; e (e) menor fecundidade entre as regiões administrativas de São Paulo em $2001(1,55)$ e imigração por idade correspondente ao saldo migratório feminino paulista observado. Os cenários (b) e (c) e os (d) e (e) permitem comparar a estrutura etária da população estável, resultante da constância da fecundidade abaixo da reposição, em dois níveis diferentes com as populações estacionárias via imigração com os mesmos níveis de fecundidade. Estas, por sua vez, podem ser comparadas à estrutura etária da população estacionária pelo aumento da fecundidade até o nível de reposição.

Apresenta-se, a seguir, o modelo teórico sobre população estacionária por meio da migração, desenvolvido por Schmertmann (1992). Com isto, explicitam-se os elementos fundamentais da inter-relação entre migração e fecundidade abaixo da reposição na trajetória rumo à estacionariedade e, comparativamente, as estruturas etárias derivadas das mudanças de parâmetros de migração e fecundidade.

\footnotetext{
${ }^{1}$ Neste método, a emigração é incorporada na projeção da mesma forma que a mortalidade, pois os dois eventos são fontes de decremento. Neste caso, de posse da informação pertinente, estimam-se as taxas de emigração e constróise uma tabela de sobrevivência de duplo decremento, para os riscos de morte e emigração. Para os fins da projeção, utilizam-se as razões de sobrevivência obtidas a partir desta tabela (PRESTON et al., 2001).

2 Ver Camargo e Yazaki (2002).
} 


\section{Modelo teórico ${ }^{3}$}

Lotka desenvolveu o modelo de população estável pressupondo população fechada à migração. No entanto, desde que a função de taxas específicas de migração líquida seja constante ao longo do tempo, a estabilidade é alcançada também no caso de populações abertas. É preciso apenas que a função de probabilidades de sobrevivência (probabilidades estas que representam as mudanças proporcionais nos tamanhos das coortes entre o nascimento e a idade a) inclua também os efeitos da migração. Se tanto as taxas de mortalidade quanto as taxas líquidas de migração forem constantes ao longo do tempo, a função de probabilidade de sobrevivência também será constante e a população tornar-se-á estável (PRESTON et al., 2001).

Nesta direção, uma série de trabalhos demonstrou que qualquer população com taxas de fecundidade constantes e abaixo da reposição e um número anual constante de imigrantes com estrutura etária fixa torna-se estacionária no decorrer do tempo (ESPENSHADE et al., 1982). Em outras palavras, assim como um aumento da fecundidade para o nível de reposição, a imigração constante bloqueia, no longo prazo, o declínio da população. Entretanto, qualquer população tornada estacionária via migração terá uma estrutura etária mais envelhecida se comparada àquela tornada estacionária pelo aumento da fecundidade até o nível de reposição (SCHMERTMANN, 1992).

O processo mais conhecido que leva uma população a se tornar estacionária implica um número anual de nascimentos (entradas) constante, taxas específicas de mortalidade (saída) constantes e que todas as entradas ocorram à idade zero (nascimentos). Este é, na verdade, um caso específico de um processo mais geral. As entradas, desde que sejam constantes, podem ocorrer em qualquer idade, ou seja, dado um conjunto de taxas de saída, existe um número infinito de populações estacionárias equivalentes, uma para cada estrutura etária de entrada.

Suponhamos a ocorrência de uma entrada anual na população à idade a. Entre este grupo, uma fração I(x)/I(a) sobreviverá até $a$ idade $x$ e(a); o tempo médio restante até a idade de saída entre aquelas com idade a no momento da entrada será dado por:

$$
e(a)=\int_{a}^{w} \frac{l x}{l a} d x
$$

O número anual de nascimentos pelo influxo de entradas anuais à idade $a, v(a)$, isto é, o número esperado de filhas nascidas após a idade a será dado por:

$$
v(a)=\int_{a}^{w} f(x) \frac{l x}{l a} d x
$$

onde $f(x)$ é a fecundidade à idade $x$.

No longo prazo, o número esperado de saídas acima da idade a será igual a 1 para cada entrada. Portanto, a cada entrada anual à idade a corresponderá exatamente uma saída. Logo, a população total será composta de subpopulações estacionárias resultantes das entradas a cada idade $a$.

Analogamente à equação $P=N^{*} e(0)$, dado um total $K$ de entradas a cada ano, o tamanho da população estacionária total será dado por $P=K^{\star} e^{\prime}$, onde $e^{\prime}$ representa, para uma entrada qualquer, o número esperado de anos vividos na população até a sua saída. O número anual de nascimentos nesta população estacionária será dado por $N=K^{*} v^{\prime}$, onde $v^{\prime}$ é o número esperado de nascimentos, para uma entrada qualquer, até a sua saída. Portanto, a Taxa Bruta de Natalidade será $n=v^{\prime} / e^{\prime}$ e a taxa de saída será dada por $o=1 / e^{\prime}$, analogamente a $n=0=1 / e(0)$ quando todas as entradas se dão pelo nascimento, a fecundidade é de reposição $\left(v^{\prime}=1\right)$ e todas as saídas se dão somente por morte.

Deve-se considerar que, numa população com fecundidade abaixo da reposição, com um conjunto de taxas específicas de mortalidade constantes ao longo do tempo e com uma entrada anual constante de

\footnotetext{
${ }^{3}$ Para efeitos deste exercício, apresentam-se apenas as relações relevantes ao processo de estacionariedade via migração presentes no trabalho deste autor.
} 
imigrantes, $I$, as entradas são de dois tipos: à idade zero (nascimentos) e à idade na imigração. No longo prazo, trata-se de duas subpopulações: imigrantes e naturais. A primeira torna-se estacionária pelo fato de que a cada entrada corresponderá uma saída. Considere-se que um ano depois de iniciada a imigração, a população de imigrantes consistirá de uma coorte de imigrantes. Dez anos depois, ela será formada pelos sobreviventes de dez coortes de imigrantes, com os sobreviventes da primeira coorte dez anos mais velhos, os da segunda coorte, nove anos mais velhos e assim por diante. Este processo segue até a morte do último sobrevivente da primeira coorte de imigrantes, momento a partir do qual a população de imigrantes torna-se estacionária. A população de naturais torna-se estacionária por meio do número constante de nascimentos. Vale lembrar que o decremento também pode ser por emigração, a qual pode ser incorporada na função de sobrevivência.

No caso da população de imigrantes, o seu total, no longo prazo, será dado por $P_{i}=$ $I^{\star} e^{\prime}{ }_{j}$ e o número anual de nascimentos será dado por $N_{i}=I^{*} v^{\prime}$. As taxas brutas de entrada (nascimentos) e saída (óbitos) serão, respectivamente, $n_{i}=v_{i}^{\prime} / e_{i}^{\prime}$ e $o_{i}=1 / e^{\prime}$. Por definição, os naturais só entram na população em questão via nascimento. Neste caso, devem ser consideradas, também, as filhas das imigrantes e suas descendentes. Se o número esperado de nascimentos anuais de filhas de imigrantes é $v_{i}$ e a Taxa Líquida de Reprodução (TLR) é menor que 1 para todas as gerações de descendentes de naturais, o número esperado de nascimentos das naturais descendentes das imigrantes convergirá, no longo prazo, para $v_{i}^{\prime} /(1-\mathrm{TLR})$ e o total de nascimentos na população estacionária será $N=/^{*} v_{i}^{\prime} /(1-T L R)$.

Se tomarmos, aleatoriamente, qualquer mulher natural da população em questão, sua entrada terá sido, por definição, à idade 0 , de forma que $e_{n}^{\prime}=e(0)$ e $v_{n}^{\prime}=$ TLR. Assim, temos que:

$P_{n}=N * e(0)=\frac{I * v_{i}^{\prime}}{1-T L R} * e(0)$ é o tamanho da população estacionária de naturais;
$n_{n}=\frac{v^{\prime}}{e^{\prime}}=\frac{T L R}{e(0)}$ é a Taxa Bruta de Natalidade da população estacionária de naturais; e

$$
o_{n}=\frac{1}{e^{\prime}}=\frac{1}{e(0)} \text { é a Taxa Bruta de Mortalida- }
$$

de da população estacionária de naturais.

Dado que a TLR $<1$, então $n_{n}<o_{n}$. No entanto, a população de naturais permanece estacionária, pois as filhas das imigrantes entram na população a uma taxa igual a $N_{i} / P_{n}$, ou:

$$
\frac{N_{i}}{P_{n}}=\frac{I * v_{i}^{\prime}}{\frac{I * v_{i}^{\prime} * e(0)}{1-T L R}}=\frac{1-T L R}{e(0)},
$$

a qual, por sua vez, corresponde à diferença entre óbitos e nascimentos de naturais:

$$
o_{n}-n_{n}=\frac{1}{e(0)}-\frac{T L R}{e(0)}=\frac{1-T L R}{e(0)} .
$$

\section{Metodologia}

Como visto na seção anterior, o arcabouço teórico desenvolvido para a análise dos efeitos da migração sobre a estrutura etária de uma população com fecundidade constante e abaixo da reposição pressupõe, como ponto de partida, duas formas de entrada - nascimento e imigração - e uma forma de saída - óbitos. Toma-se o conjunto das taxas específicas de mortalidade como constante e um número de entradas de imigrantes, por idade, também constante. Além disso, pressupõe-se que as imigrantes experimentam as mesmas condições de fecundidade e mortalidade das naturais.

Neste exercício, utiliza-se o método dos componentes para projetar a estrutura etária futura em cada cenário estipulado, dentro dos pressupostos mencionados anteriormente. $\mathrm{O}$ grupo populacional relevante para efeitos do exercício é a população feminina do Estado de São Paulo em 2000. O conjunto de taxas específicas de fecundidade empregado é aquele observado em 2000 , correspondente a uma Taxa de Fecundidade Total (TFT) de 2,04 (IBGE, 2000), ajustadas para os níveis de fecundidade total utilizado em cada cenário. O número de nascimentos esperado em cada período de projeção é obtido utilizando-se 
as taxas específicas de fecundidade para os respectivos grupos qüinqüenais. Os nascimentos são distribuídos por sexo, de acordo com a razão de sexo ao nascer usualmente utilizada, de 1,05. A tábua de vida empregada representa a experiência de mortalidade relativa ao meio do decênio 1990-2000, implicando uma esperança de vida ao nascer para as mulheres de 73,7 anos. ${ }^{4}$

Com relação à migração, o número de imigrantes e sua estrutura etária utilizada, como mencionado, correspondem ao saldo migratório feminino, por grupos de idade, observado no qüinqüênio 1995-2000 entre o Estado de São Paulo e o restante do país. Para efeitos deste exercício, estes valores foram tomados apenas como parâmetros e a migração internacional não é levada em conta, ou seja, pressupõe-se um volume de imigrantes por idade correspondente aos saldos da migração interna. No caso dos grupos etários que apresentaram saldo negativo, tomou-se uma entrada de imigrantes igual a zero. A Tabela 1 mostra a distribuição do saldo migratório interno feminino relativo ao Estado de São Paulo, segundo grupos etários.
Os cenários de simulação efetuados foram os seguintes:

(a) fecundidade ao nível de reposição e ausência de imigração: este cenário estabelece a estrutura etária mais jovem possível, ou seja, a população tornada estacionária por entradas constantes à idade zero;

(b) TFT observada em 2000 (2,04) e imigração por idade correspondente ao saldo migratório feminino paulista observado no qüinqüênio 1995-2000: este cenário aponta o resultado da estabilização da fecundidade no nível observado em 2000 e fornece uma indicação da composição por idade da população paulista tornada estacionária via migração, sob o pressuposto de que a mortalidade e a fecundidade permanecessem constantes ao longo do tempo;

(c) fecundidade em 2000 (2,04) e imigração concentrada no grupo etário 15-19: este cenário reproduz a estrutura etária da população estacionária caso todas as imigrantes

TABELA 1

Saldo migratório feminino, segundo grupos etários Estado de São Paulo - 1995-2000

\begin{tabular}{cc}
\hline Grupos etários & Saldo migratório \\
\hline $0-4$ anos & 5.181 \\
$5-9$ anos & 10.790 \\
$10-14$ anos & 16.199 \\
$15-19$ anos & 57.922 \\
$20-24$ anos & 95.503 \\
$25-29$ anos & 46.216 \\
$30-34$ anos & 13.241 \\
$35-39$ anos & 2.305 \\
$40-44$ anos & -589 \\
$45-49$ anos & 13 \\
$50-54$ anos & -1.458 \\
$55-59$ anos & -837 \\
$60-64$ anos & -304 \\
$65-69$ anos & -342 \\
$70-74$ anos & -230 \\
$75-79$ anos & 260 \\
$80-84$ anos & 524 \\
\hline anos e mais & 309 \\
\hline
\end{tabular}

Fonte dos dados básicos: IBGE, Censo Demográfico 2000, microdados da amostra. Cálculos elaborados pelo autor.

\footnotetext{
4 Comunicação informal (não publicada), gentilmente cedida por WONG, L. L. R.. Tábuas de vida para o estado de São Paulo 1990-2000.
} 
passassem a residir no estado entre 15 e 19 anos de idade. Esta hipótese "heróica" visa demonstrar o efeito sobre o tamanho e a estrutura etária da população de destino se as imigrantes experimentassem toda a sua vida reprodutiva no destino. Analogamente, pode-se pensar que o efeito da fecundidade das imigrantes seria nulo se todas chegassem ao destino com 50 anos de idade;

(d) menor fecundidade observada entre as regiões administrativas de São Paulo em 2001 (1,55, na RA de São José do Rio Preto) e imigração por idade correspondente ao saldo migratório feminino paulista observado: este cenário fornece o efeito da migração sobre a estrutura etária da população estacionária via imigração paulista, na hipótese de as TFTs de suas regiões administrativas convergirem para o nível mínimo observado no estado. Este cenário implica menores fecundidade e efeito da fecundidade das imigrantes na população de destino, se comparado ao cenário seguinte;

(e) menor fecundidade observada entre as regiões administrativas de São Paulo em 2001 (1,55, na RA de São José do Rio Preto) e imigração concentrada no grupo etário 15-19: como em (c), este cenário gera uma população estacionária via migração, a mais jovem possível.

A análise dos resultados é realizada por meio dos seguintes indicadores: (1) comparação da estrutura etária final implícita em cada uma das populações estacionárias geradas - cenários (a) a (e) com a estrutura etária inicial (Estado de São Paulo, ano 2000); (2) idade média implícita nestas estruturas etárias; (3) relação entre o tamanho de cada uma das populações estacionárias finais e a estrutura etária inicial; (4) razão de suporte potencial (RSP), que é a razão entre as populações de 15 a 64 anos em relação àquela de 60 anos e mais; (5) tamanho da população estacionária final em relação ao tamanho da população inicial.

\section{Resultados}

Na Tabela 2, apresentam-se as distribuições etárias da população inicial e das estacionárias derivadas das simulações.

Em termos gerais, a estrutura etária da população inicial é composta por pouco mais de um quarto de mulheres com menos de 15 anos. Nas simulações realizadas, a maior proporção foi, no máximo, de 19,3\% (cenário (a)). Do mesmo modo, a proporção de indivíduos nas idades de 15 a 64 anos foi máxima na população inicial, atingindo o valor de $64,2 \%$ no cenário (e). Finalmente, nas idades acima de 65 anos, a proporção na população inicial foi de menos de $7 \%$, enquanto nas simulações o valor mínimo obtido foi de $19,9 \%$ (cenário (a))

Os resultados da Tabela 3 confirmam os achados da Tabela 2, no que tange à idade média e à razão de suporte potencial, uma vez que a menor idade média é a da população inicial, inferior a 31 anos $(30,9)$, e a menor, entre as simulações realizadas, é a do cenário (a), de 40,6 anos, ou seja, 0,7 ano superior. No que se refere à razão de suporte potencial, enquanto a da população inicial é de 9,8 pessoas das idades 15 a 64 anos em relação às de 65 anos e mais, a maior razão, entre os cenários, é a do cenário (a), de 3,1, ou seja, a da população inicial é mais de 2

TABELA 2

Estrutura etária da população inicial e das populações estacionárias Estado de São Paulo - 2000

\begin{tabular}{lcccccc}
\hline Grupos etários & População inicial & Cenário (a) & Cenário (b) & Cenário (c) & Cenário (d) & Cenário (e) \\
\hline 0-14 anos & 0,254 & 0,193 & 0,182 & 0,183 & 0,142 & 0,145 \\
$15-64$ anos & 0,677 & 0,608 & 0,615 & 0,615 & 0,638 & 0,642 \\
65 anos e mais & 0,069 & 0,199 & 0,203 & 0,202 & 0,220 & 0,213 \\
Total & $\mathbf{1 , 0 0}$ & $\mathbf{1 , 0 0}$ & $\mathbf{1 , 0 0}$ & $\mathbf{1 , 0 0}$ & $\mathbf{1 , 0 0}$ & $\mathbf{1 , 0 0}$ \\
\hline
\end{tabular}

Fonte dos dados básicos: IBGE, Censo Demográfico 2000, microdados da amostra. Cálculos elaborados pelo autor. 
vezes maior Finalmente, com respeito ao tamanho da população estacionária final em relação ao tamanho inicial, observa-se que, no cenário (a), este aumento é de $20 \%$ em relação ao inicial, atingindo seu máximo no cenário (c), em que a população estacionária final passa a ser 3,1 vezes a inicial. Esta relação atinge seu mínimo no cenário (d), na qual a população estacionária final passa a ser $47 \%$ menor relativamente à inicial.

TABELA 3

Razão de tamanho da população final em relação à inicial, idade média e razão de suporte potencial, segundo população inicial e cenários de (a) a (e)

Estado de São Paulo - 2000

\begin{tabular}{lr}
\hline População & Resultados \\
\hline População Inicial & 1,1 \\
Tamanho da população final em relação à inicial & 30,9 \\
Idade média & 9,8 \\
Razão de suporte potencial & 1,2 \\
Cenário (a) & 40,6 \\
Tamanho da população final em relação à inicial & 3,1 \\
ldade média & \\
Razão de suporte potencial & 2,4 \\
Cenário (b) & 41,3 \\
Tamanho da população final em relação à inicial & 3,0 \\
Idade média & \\
Razão de suporte potencial & 3,1 \\
Cenário (c) & 41,1 \\
Tamanho da população final em relação à inicial & 3,0 \\
Idade média & \\
Razão de suporte potencial & 0,53 \\
Cenário (d) & 43,7 \\
Tamanho da população final em relação à inicial & 2,9 \\
Idade média & \\
Razão de suporte potencial & 0,64 \\
Cenário (e) & 42,9 \\
Tamanho da população final em relação à inicial & 3,0 \\
ldade média & \\
Razão de suporte potencial & \\
\hline
\end{tabular}

Fonte dos dados básicos: IBGE, Censo Demográfico 2000, microdados da amostra. Cálculos elaborados pelo autor.

\section{GRÁFICO 1}

Estrutura etária das populações estacionárias equivalentes, segundo cenários Estado de São Paulo - 2000

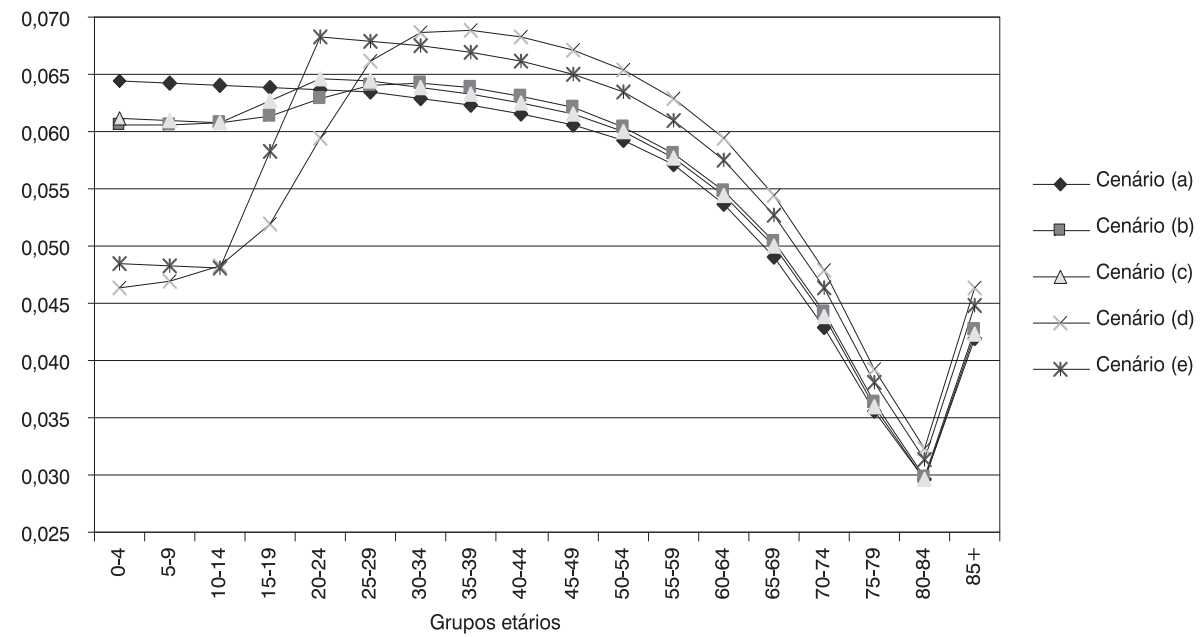

Fonte dos dados básicos: IBGE, Censo Demográfico 2000, microdados da amostra. Cálculos elaborados pelo autor. 


\section{Considerações finais}

Os resultados das simulações indicam um aumento inequívoco da proporção da população acima de 65 anos, aliado ao declínio da proporção abaixo de 15 anos, implicando uma razão de suporte potencial de, no máximo, 3,1. Em relação especificamente aos cenários criados, aqueles nos quais as imigrantes se concentram no grupo etário 15 a 19 anos - cenários (c) e (e) - tendem a rejuvenescer a população, relativamente aos (b) e (d), respectivamente. De fato, a situação mais extrema se refere ao cenário (d), no qual a população estacionária equivale a praticamente a metade da inicial, tem a maior idade média entre todos os cenários e razão de suporte potencial no nível dos demais cenários.

Este exercício é indicativo da importância da migração no jogo, no longo prazo, dos componentes demográficos em uma população com Taxa Líquida de Reprodução abaixo da unidade. Deve-se considerar que, no caso do retorno à estacionariedade via migração, a estrutura etária das entradas é fundamental, como denota o cenário (e). Por outro lado, o nível de fecundidade atingido é o fator de maior peso, pois o retorno à estacionariedade via aumento da fecundidade - cenário (a) - é o processo de efeitos menos intensos em relação ao decréscimo da população e ao seu envelhecimento.

Este exercício é, por estes motivos, sugestivo da dimensão dos dilemas e desafios que a dinâmica demográfica do país já impõe - e imporá com maior intensidade - à sociedade brasileira. Por um lado, a Taxa de Fecundidade Total, calculada seja por meio dos dados da Pesquisa Nacional por Amostra de Domicílios (PNAD) 2007, seja a partir das informações coletadas pela Pesquisa Nacional de Demografia

\section{Referências bibliográficas}

CAMARGO, A. B. M.; YAZAKI, L. M. A fecundidade recente em São Paulo: abaixo do nível de reposição? In: ENCONTRO DE ESTUDOS POPULACIONAIS, XIII. Anais... Ouro Preto, Abep, 2002. e Saúde (PNDS) 2006, respectivamente 1,95 e 1,8, coloca o Brasil em um patamar pós-transicional, no qual é factível supor a continuidade da queda. Por outro lado, não há indícios de que o país venha a se tornar um destino de fluxos migratórios internacionais de monta. Pelo contrário, os saldos calculados tendem a ser negativos (CARVALHO; CAMPOS, s.d.). Ainda que em prazo bastante estendido, o cenário mais plausível, atualmente, para o Brasil do futuro é uma situação similar ao cenário (d), isto é, ausência de fluxos migratórios substanciais em direção ao país e fecundidade abaixo dos níveis encontrados em 2006 e 2007, ou seja, diminuição substancial da população, seu envelhecimento intenso e razões de suporte potencial altas.

É importante frisar que este exercício configura-se como uma simulação e não como uma projeção. Não se objetivou, de forma alguma, prever a estrutura etária da população de São Paulo no futuro. Mesmo por que os pressupostos de permanência dos naturais e dos imigrantes, de constância das taxas de entrada e saída e do número de imigrantes por idade, bem como a incorporação das experiências de mortalidade e fecundidade das naturais pelas imigrantes, a partir do momento de entrada, não são exatamente realistas. Entretanto, para se analisarem os efeitos da plasticidade dos fenômenos demográficos, é preciso estabelecer elementos de comparação. Assim, o que se almejou foi demonstrar, por meio de um modelo teórico, as implicações de longo prazo derivadas da manutenção de parâmetros demográficos observados no curto prazo e identificar os efeitos de mudanças em um componente (migração) sobre a estrutura etária da população no longo prazo. Neste aspecto, a conexão entre migração e fecundidade não deve ser olvidada.

CAMPOS, N. O. B.; RODRIGUES, R. N. Ritmo de declínio nas taxas de mortalidade dos idosos nos estados do Sudeste, 1980-2000. Revista Brasileira de Estudos de População, Abep, v. 21, n. 2, p. 323-342, 2004. 
CARVALHO, J. A. M.; CAMPOS, M. B. A variação do saldo migratório internacional do Brasil entre as duas últimas décadas: uma indicação de diminuição significativa do fluxo de emigrantes brasileiros, s.d. Mimeografado.

ESPENSHADE, T. J.; BOUVIER, L. F.; ARTHUR, W. B. Immigration and the stable population model. Demography, v. 19, n. 1, p. 125-133, 1982.

FEICHTINGER, G.; STEINMANN, G. Immigration into a population with fertility below replacement level - the case of Germany. Population Studies, v. 46, p. 275-284, 1992.

MITRA, S. Generalization of the immigration and the stable population model. Demography, v. 20 , n. 1, p. 111-115, 1983.

MITRA, S. Immigration, below-replacement fertility, and long-term national population trends. Demography, v. 27, n.1, p. 121-129, 1990.

NAÇÕES UNIDAS - Economic and Social Affairs/Population Division. Replacement migration: is it a solution for declining and ageing population? ESA/P/WP 160. Nova Iorque, Nações Unidas, 2000. Disponível em: <http://www.un.org/esa/population/ publications/migration/migration.htm $>$. Acesso em: 9 set. 2008.

PRESTON, S. H.; HEUVELINE, P.; GUILLOT, $M$. Demography: Measuring and modeling population processes. Oxford: Blackwell Publishers, 2001.

SCHMERTMANN, C. P. Immigrants' ages and the structure of stationary populations with below-replacement fertility. Demography, v. 29, n. 4, p. 595-612, 1992

\section{Resumen}

Fecundidad por debajo de la reposición, población estacionaria por migración y efectos sobre la estructura etaria

Ante el declive de la fecundidad, Brasil se aproxima a las condiciones demográficas que determinan el crecimiento cero o negativo, en el largo plazo, de una población. Entretanto, no hay razones suficientes para afirmar que la fecundidad se estabilizará en el nivel de reposición o un poco por debajo de él. Hay indicaciones de que ésta debe alcanzar cifras bastante bajas a ese nivel en las próximas décadas. Teóricamente, una población estable y cerrada con tasas de fecundidad por debajo del nivel de reposición puede retomar el crecimiento cero si es sometida a un cambio del número anual constante de entradas derivadas del retorno de la fecundidad al nivel de reposición. Esto también puede ocurrir debido a saldos netos migratorios positivos, constantes y con estructura etaria fija. Por lo tanto, para un determinado conjunto de tasas de salida existe un número infinito de poblaciones estacionarias equivalentes. Presuponiendo un régimen de fecundidad por debajo de la reposición, este trabajo utiliza el modelo teórico de Schmertmann (1992) para simular y examinar, comparativamente, la estructura etaria de la población del Estado de San Pablo, resultante de un aumento de la fecundidad hasta el nivel de reposición, y las estructuras etarias de esta misma población tornada estacionaria vía migración, asumiendo diferentes escenarios de fecundidad y estructura etaria de las inmigrantes.

Palabras-clave: Migración. Fecundidad por debajo de la reposición. Estructura etaria. Envejecimiento poblacional. 


\begin{abstract}
Fertility below replacement, stationary population through migration and effects of age structures

Due to its declining fertility rate, Brazil is closer to the demographic conditions that determine population zero or negative growth, on the long run. However, there are no reasonable grounds to assert that the fertility rate will stabilize at replacement level or just below it. There are indications that it will be likely to descend to very low levels in coming decades. Theoretically, a stable and closed population with fertility rates below replacement level can return to zero growth if the population is subjected to changes in the constant annual number of entries derived from the return of fertility to replacement level. This may also occur due to constant positive net migration with constant and fixed age structure. Therefore, for a given set of rates of exits, there are an infinite number of equivalent stationary populations. Given fertility below replacement level, the present study uses Schmertmann's theoretical model (1992) to simulate and examine, by comparison, (1) the age structure of the population of the State of São Paulo, Brazil, that has resulted from a rise in fertility to replacement level and (2) the age structures of this population, as made stationary through migration, assuming different scenarios of fertility and the age structure of immigrants.
\end{abstract}

Keywords: Migration. Below replacement fertility. Age structure. Ageing of population.

Recebido para publicação em 05/09/2008. Aceito para publicação em 14/11/2008. 